\title{
Malaria Outbreak Facilitated by Increased Human Activities around Swamps: Kisoro District, Uganda, December 2017-March 2018
}

Joyce Nguna ( $\sim$ joynguna@musph.ac.ug )

Makerere University

Dennis Okethwangu

Makerere University

Daniel Kadobera

Makerere University

Research article

Keywords: Malaria, Disease Outbreaks, Global Health Security, Uganda

Posted Date: July 4th, 2019

DOl: https://doi.org/10.21203/rs.2.10936/v1

License: @ (i) This work is licensed under a Creative Commons Attribution 4.0 International License. Read Full License 


\section{Abstract}

Background: Malaria elimination is increasingly becoming a global priority. On 19 December 2018, a district in southwestern Uganda known to have achieved pre-elimination levels of malaria (<30 cases per month) reported a sudden increase in cases to the Ministry of Health. We investigated to determine the magnitude and scope of the outbreak and identify exposures associated with transmission. Methods:We reviewed medical records in all health facilities in the affected Kanaba and Murora sub-counties to find cases. We calculated attack rates (AR) by age, sex, and village using the projected 2016 population. In a case-control study, we compared potential exposures between case-patients, selected randomly from the line-list, and neighborhood- and age-matched asymptomatic controls. We conducted an entomological and environmental assessment of randomly-selected households and potential breeding sites of the affected sub-counties. Results: We found 3,130 malaria cases (compared with 879 cases during the same period the previous year). Persons in the age groups 10-19 $(A R=14 / 1,000)$ and 20-29 (AR=12/1,000) years were the most affected. Villages closest to the swamp had the highest attack rate. In the case-control study, 89\% (129/145) of case-patients and 73\% (106/145) of controls reported frequent late-night mosquito bites (ORCLR=3.9; 95\%Cl: 1.8-8.4); 70\% (102/145) of case-patients and $59 \%(86 / 145)$ of controls had a household size $>5$ (ORCLR $=1.8 ; 95 \% \mathrm{Cl}$ : $1.04-3.1) ; 78 \%(113 / 145)$ of case-patients and $86 \%(125 / 145)$ of controls usually slept under a bednet (ORCLR=0.56; 95\%Cl: 0.29-1.1). Anopheles gambiae sensu lato was identified in breeding places around Sereri and Mpundu swamps. In total, 64\% (23/36) of female adult Anopheles captured in case-patients' households by pyrethrum spray catches were fed, suggesting low bednet usage the previous night in the surveyed households. Conclusion: This malaria outbreak was likely propagated by favorable breeding conditions, including recent heavy rainfall, and exacerbated by new human activities around two swamps. To achieve malaria elimination, low-endemic areas should be particularly aware of activities that can lead to resurgences in malaria, such as night-time exposures, lack of bed-net usage, and human activities focused around breeding sites. We recommended increased awareness about bed-net usage and use of larvicides in the residential area and swamps to break the breeding cycle.

\section{Introduction}

Malaria is a life-threatening febrile illness caused by Plasmodium parasites that are transmitted to people through bites of infected Anopheles mosquitoes[1]. Five parasite species cause malaria in humans; $P$. falciparum and $P$. vivax pose the greatest threat[1]. Sub-Saharan Africa, where $P$. falciparum is the predominant species, has the highest malaria disease burden of any region worldwide. Malaria is also re-emerging in most of the African highlands, exposing non-immune populations to deadly epidemics [2]. Typically, malaria has been associated with poor housing and infrastructure, and travel to rural areas [3]. However, climatic and environmental changes can lead to surges or resurgences in areas that normally experience only intermittent or low-grade transmission $[4,5]$.

Despite progress in recent years in scaling up malaria control interventions in Sub-Saharan Africa and elsewhere, malaria remains one of the most important global health challenges, accounting for significant morbidity and mortality [6, 7]. The global focus on malaria is now shifting away from smaller-scale control towards efforts to eliminate and eventually eradicate malaria [9]. Malaria elimination involves reducing to zero the incidence of infection caused by human malaria parasites in a defined geographical area, through deliberate efforts. 'Preelimination' consists of the period of reorientation of malaria control programmes between the sustained control 
and elimination stages, when coverage with good-quality laboratory and clinical services, reporting, and surveillance are reinforced, followed by other programme adjustments to halt transmission nationwide [8].

In Uganda, the climate allows stable, year-round malaria transmission with relatively little seasonal variability in most areas. Ninety-five percent of the population in Uganda resides in areas where malaria is highly endemic; malaria is the leading cause of healthcare utilization in the country [9]. The epidemiology of malaria varies widely in Uganda, from highland regions with low prevalence and intermittent transmission to regions of dense agricultural settlement where the transmission intensities are among the highest in the world [10]. The Ugandan Ministry of Health (UMOH), with several implementing partners, have supported various strategies for malaria control, including enforcing adherence to national case management guidelines for mild and severe malaria at health facilities and communities, distribution of long-lasting insecticide-treated nets, indoor residual spraying, and prevention of malaria in pregnancy [11-13]. This has led to a decline in national malaria prevalence from 45\% in 2010 to 19\% in 2016 [14, 15]. However, most areas of Uganda are still highly endemic, and not approaching elimination of malaria.

Kisoro District is located in a highland area in southwestern Uganda, and typically has low malaria transmission (malaria parasite prevalence of $12 \%$, compared with the national average of $19 \%$ [9]). As such, the population is non-immune and susceptible to epidemics. On 19 December 2018, a malaria outbreak was reported in two of the sub-counties of Kisoro District, Kanaba and Murora sub-counties. The outbreak was detected through routine analysis of malaria surveillance data reported in the District Health Information System 2 (DHIS2) for December 2017 (weeks 49-52, 2017) and January 2018 (weeks 1-2, 2018) [16]. This analysis showed a sudden increase in malaria cases (>200 cases/month from DHIS2 during this time period) compared to the previously-reported cases from DHIS2 [16]. On the contrary, two subcounties of Muko and Bufundi in Rubanda district, neighbouring the affected subcounties of Kanaba and Murora, recorded lower malaria cases (65-80 cases/month) for the same reporting period, i.e., December 2017 (weeks 49-52, 2017) and January 2018 (weeks 1-2, 2018) [17]. Cases diagnosed by malaria rapid diagnostic test or microscopy in the district in this time period exceeded the action thresholds established by UMOH's Malaria Control Program [16]. We investigated this outbreak to determine its magnitude and scope, identify factors associated with transmission in this normally low-endemic area, and recommend evidence-based control and prevention measures.

\section{Methods}

\section{Study area}

Kisoro District (Coordinates at center of district town: Latitude: -1.28538; Longitude: 29.68497; elevation: 1,890m) is in southwestern Uganda. The climate is typically cool with average ambient temperatures between $17.2^{\circ} \mathrm{C}$ (low) and $27^{\circ} \mathrm{C}$ (high), and an average annual rainfall of 1,368 $\mathrm{mm}$. Kisoro District has 14 sub-counties with an estimated population of 301,617 in 2014 [18]. The population is served by 39 health facilities (including 2 hospitals and 37 lower-level health facilities), all with the capacity to test for and treat malaria. Farmers in the rural Kanaba and Murora sub-counties, where the outbreak occurred, grow Irish potatoes in the highlands and swamps as their main agricultural activity.

\section{Case definition and finding}


We defined a case as a positive malaria rapid diagnostic test (mRDT) or microscopy from 19 December 2017 to 30 March 2018 in a resident or visitor of Kanaba and Murora sub-counties in Kisoro District.

We reviewed data in DHIS2 on malaria cases reported by the health facilities serving the affected sub-counties [16]. We also systematically searched for malaria cases by reviewing health records from 19 December 2017 to March 2018 in health facilities in the 2 most affected sub-counties, Kanaba and Murora. We reviewed outpatient, inpatient, and laboratory records from all 4 health facilities in the outbreak-affected area. Patient records contained basic information (i.e., name, age, sex, and place of residence), as well as date of diagnosis and admission.

\section{Descriptive epidemiology}

We computed the attack rate (AR) by age, sex, and sub-county of residence, using population data from the Uganda population and housing census data projections for 2017 [18]. We constructed an epidemic curve to describe the distribution of cases over time. We used the scatter plot to evaluate the relationship between the attack rates in the villages and their approximate distance to the nearest swamp.

\section{Hypothesis generation}

Using a standardized questionnaire, we interviewed a random sample of 30 confirmed case-patients on their exposures, including not sleeping under a bed-net, going to bed after 9:00 pm, previous history of malaria, not wearing long sleeves or long pants in the evening, and living near a swamp during the effective exposure period (based on the incubation period for malaria).

\section{Case-control study}

To test the hypotheses generated, we conducted a 1:1 matched case-control study. We selected cases from the line-list using systematic random sampling. If a household had more than one case selected, we only enrolled the case-patient with the earliest date of onset. We selected the controls who did not have any malaria symptoms from 19 December 2017 to the interview date among residents in the same neighborhood as the case-patient, matched by age group. Using StatCalc in Epi Info 7, considering a power of $80 \%$, two-sided confidence level of $95 \%$, a case-control ratio of $1: 1$ with $75 \%$ of cases exposed and $85 \%$ of controls exposed, we calculated a sample size of 142 cases and 142 controls. To account for potential non-response, we inflated the number to 150 cases and 150 controls.

For each case-control pair, we assessed exposures during the case-patient's effective exposure period, defined as the time window between the minimum and maximum incubation period (i.e., 7-14 days before the case-patient's onset). We collected data from both the case-patients and controls on their demographics, as well as whether or not the respondents owned and used a bed-net, lived near a swamp, and participated in late outdoor activities such as cooking, attending to shops or vending stands, or socialising. We also collected the Global Positioning System (GPS) coordinates of case-patients' place of residence. 
To account for the matched study design in the case-control study, we used conditional logistic regression to calculate the odds ratio $\left(\mathrm{OR}_{\mathrm{CLR}}\right)$ and $95 \%$ confidence interval $(95 \% \mathrm{Cl})$, with the match set as the matching variable.

\section{Entomological assessment and testing}

We collected adult mosquitos using Pyrethrum Spray Catches in the three villages of Mpundu, Butoke, and Kooga that had high malaria attack rates during the outbreak. We selected 3 houses from each village. During early morning hours, indoor resting mosquitoes were knocked down onto white ground sheets using an aerosol pyrethrum insecticide and collected using forceps and petri dishes. We identified adult mosquitoes in the field using morphological characteristics under a stereomicroscope and assessed if they were blood-fed, and preserved them dry for parasitological analysis using silica gel.

We searched for breeding sites for malaria vectors in stagnant water pools within $2 \mathrm{~km}$ of the affected villages, based on the fact that the mosquito's flight range is about 2-3 km [19]. In each of the stagnant water pools, we searched for larvae using entomological scoops. We also identified human activities that could lead to the creation of breeding sites. We then characterized active and potential breeding sites. We also compared rainfall data with the number of malaria cases over the investigation period.

For environmental assessment, we observed physical features in the affected villages. We searched for factors that could be facilitating mosquito breeding, including the materials used for construction and roofing of houses.

For entomological laboratory investigation, we followed the Plasmodium falciparum Circumsporozoite (PFCS) enzyme-linked immunosorbent assay (ELISA) Protocol of the Centers for Disease Control and Prevention (CDC) [20]. This PFCS-ELISA assay detects antibody or antigen. We ensured quality control by adding a positive control and a negative control (male mosquito samples) for the test to be validated before running.

\section{Ethical considerations}

This investigation was in response to a public health emergency and was therefore determined to be nonresearch. UMOH gave the directive and approval to investigate this outbreak. The Office of the Associate Director for Science at the CDC's Center for Global Health determined that this activity was not human subjects research, and its primary intent was public health practice or a disease control activity. Verbal informed consent in the local language was sought from respondents or caretakers of diseased children. They were informed that their participation was voluntary and their refusal would not result in any negative consequences. To protect the confidentiality of the respondents, we assigned unique identifiers for participants instead of their names during data analysis.

\section{Results}

\section{Descriptive epidemiology}

During 19 December 1, 2017 to March 31, 2018, 3,130 malaria cases were reported at health facilities in the 2 most affected sub-counties, Murora and Kanaba. During the same period in the previous year, 879 cases were 
reported. The median age of case-patients was 18.8 years (range: 0-90 years); age groups 10-19 $(A R=14 / 1,000)$ and 20-39 years most affected $(A R=12 / 1,000)$ (Table 1$)$. Males $(A R=12 / 1,000)$ and females $(A R=11 / 1,000)$ were similarly affected. The outbreak affected all the villages in the 2 most affected sub-counties (Figure 1).

Chibumba village had the highest attack rate $(487 / 1,000)$, followed by Butoke $(457 / 1,000)$, Mpundu $(415 / 1,000)$, Kooga $(291 / 1,000)$, and Mukaka $(233 / 1,000)$ (Figure 2). The distribution of the cases enrolled in the case-control study, which were randomly selected from the overall line-list, showed that cases clustered around the two swamps (Figure 2). Although the large number of total cases prevented us from obtaining the GPS coordinates for all case-patients' place of residence, case-patients enrolled in the case-control study should be representative of all case-patients during the outbreak since they were selected randomly from the line-list. Villages located $<500$ meters from the nearest swamp ( $A R=275 / 1000)$ were nearly 6 times as likely to be affected as villages located $\geq 500$ meters from the nearest swamp (48/1000) (RR=5.8, 95\% Cl: 5.3-6.3) (Figure 3).

The epidemic curve indicates continuous, gradual build-up of cases that started in November 2017, after a series of light to moderate rainfalls. During the week of December 17, 2017, the number of cases exceeded the outbreak threshold. Subsequently, cases continued to rise and peaked during the week of January 21, and declined afterwards. By the week of April 1, 2018, the case count had returned to the non-outbreak level (Figure 4).

\section{Hypothesis generation findings}

Of the 30 case-patients interviewed, $80 \%$ (24/30) reported not sleeping under a bed-net, $73 \%$ (22/30) reported often going to bed after $9: 00 \mathrm{pm}, 67 \%(20 / 30)$ had history of a malaria-infected person in the household during the outbreak period, $67 \%(18 / 30)$ were living near a swamp, and $63 \%(19 / 30)$ reported that they usually wore long clothes in the evening. We used these pieces of information to develop our hypotheses and refine our questionnaire.

Case-control study results

In total, 145 case-patients and 145 controls participated in the case-control study. In the case-control study, 89\% of case-patients and $73 \%$ of controls reported having frequent late-night mosquito bites before going to bed $\left(\mathrm{OR}_{\mathrm{CLR}}=3.9 ; 95 \% \mathrm{Cl}: 1.8-8.4\right) ; 70 \%$ of case-patients and $59 \%$ of controls had a household size $>5\left(\mathrm{OR}_{\mathrm{CLR}}=1.8\right.$; $95 \% \mathrm{Cl}: 1.04-3.1) ; 78 \%$ of case-patients and $86 \%$ of controls usually slept under a bed-net at night $\left(\mathrm{OR}_{\mathrm{CLR}}=0.56\right.$; $95 \% \mathrm{Cl}: 0.29-1.1)$. The presence of containers and/or overgrown bushes in the neighborhood was associated with malaria in an additive fashion. Living near a swamp were not significantly associated with malaria (Table 2).

\section{Environmental assessment}

Villages in the outbreak-affected sub-counties are located on hills around the Sereri and Mpundu swamps. The swamp property previously belonged to the district, and was not being used for agricultural activities until July 2017. In July 2017, residents living in the surrounding villages applied and were approved to cultivate the swamp for rice-farming as well as Irish potato-growing and brick-making.

When we examined the environment at the Sereri Trading Center, a population center at the heart of the outbreak, we found numerous pools of stagnant water in trenches of feeder roads. These pools reportedly dry up 
approximately one week after the rain stops. A large number of Anopheles larvae were found in these pools using entomological scoops.

Mud pits were created in the middle of Mpundu swamp to make bricks. These pits contained both large and small pools of stagnant water. During the study period, the small pools had a high density of young-stage Anopheles mosquitos by visual inspection; however, the densities were not quantitatively measured. Like the roadside pools, the small pools in the brick-making area dry out after a week without rain. However, the larger mud pits hold water for a longer period, and support mosquito breeding. Tire tracks on paths leading to the mud pits also provided small pools, which could support mosquito breeding if there was continuous rain.

In addition, trenches were found throughout the agricultural fields inside and outside of the swamp. When it rains, stagnant water would be held between mounds of Irish-potato and cabbage cultures, which may have created breeding sites for Anopheles. The two most-affected villages, Chibumba and Butoke, were observed to have more stagnant water pools in the agricultural fields than the less affected villages.

\section{Entomological assessment and laboratory investigation}

Anopheles gambiae sensu lato was the only species identified using the Pyrethrum Spray Catches. We sampled 12 households and identified 36 adult Anopheles mosquitoes from eight households (67\%). Of the 36 adult Anopheles caught, 23 (64\%) were fed; 16 (70\%) were gravid and 7 (30\%) were semi-gravid (i.e., not fully fed). On laboratory analysis using Pf-Circumsporozoite ELISA, none of the mosquitos were infected by the malaria parasites; however, all were nulliparous (i.e., they were young and had not lived long enough to complete the extrinsic incubation period).

\section{Discussion}

We investigated an outbreak of malaria in Kisoro District, a district that previously had low levels of malaria. The outbreak started during mid-December 2017 after a series of light to moderate precipitations that began in early November 2017 and lasted until the end of March 2018. The rainfall likely contributed to environmental conditions that favored mosquito breeding, as did the cultivation of swamps for agricultural activities, which could have increased the suitability of the reclaimed swamp for vector breeding and increased human interactions with mosquitoes. This kind of explosive outbreak after an initial generation of cases has occurred in other vector-borne disease outbreaks [21].

In addition to the above-mentioned driving forces for the outbreak, having frequent late-night mosquito bites before going to bed, presence of containers and/or overgrown bushes in the neighborhood, and large household size were significant risk factors for malaria infection during this outbreak. Usually sleeping under a bed-net had a borderline-significant protective effect against malaria. These results are consistent with findings during previous investigations $[19,22-26]$. The high percentage of fed mosquitoes in the rooms using the Pyrethrum Spray Catches indicated that the occupants of the rooms had either slept under defective bed-nets or had not used them. Environmental and entomological investigations revealed the presence of favourable breeding sites for Anopheles mosquitos due to human activity - specifically rice-farming, Irish potato-growing, and brickmaking - in the swamps. Although growing of rice and Irish potato was not new, the use of the swamp for these 
practices was only implemented in July 2017. Also, the kilns for brick-making may have created localized warmer conditions and pools of standing water that are favorable for mosquito growth.

Attack rates were extremely high in the villages surrounding the swamps, approaching $50 \%$ in several. Mpundu village, which had the highest number of cases, contains both the Mpundu-Sereri swamp and several brick pits. The two villages with the next-highest numbers of cases, Chibumba and Butoke, were similarly close to the swamp, and had large areas of cultivated swamp within their borders. These result regarding the association between proximity to breeding sites and malaria attack rate is similar to findings in other studies $[2,27]$. They are also consistent with the literature demonstrating that changes in the environmental conditions, such as expansion of rice paddy fields and other agriculture activities, may expand both the malaria-prone areas and the population at risk $[2,28]$. Such a phenomenon had come to the researchers' attention in India more than 80 years ago, and was dubbed "man-made malaria" [29]. The same problem has persisted to this day in various parts of the world[30,31]. The lack of significant association between malaria infection and self-reported "having a swamp nearby" in the case-control study likely was due to overmatching by the use of neighborhood controls, which had negated the effect.

Literature also shows that vector density, transmission rates, and malaria incidence can vary widely within small geographic areas as a result of small variations in risk factors, such as temperature, standing water, and vegetation [4-6]. Replacement of natural swamp vegetation with agricultural crops can increase the temperature in the microenvironment, leading to elevated malaria endemicity in cultivated areas [5].

Moreover, the lower number of malaria cases reported in the neighbouring Muko and Bufundi subcounties in Rubanda District could be due to the differences in the economic activities. Although both Rubanda and Kisoro districts are predominantly crop growing, there were no human activities such as brick laying in Rubanda District that created mosquito breeding sites to lead to a malaria outbreak.

During this outbreak, being older than 18 years was associated with a higher risk of malaria infection. This finding contradicts the general age distribution of malaria that children have higher incidence than adults and investigations conducted elsewhere [23-25]. The finding of higher risk in adults supports the idea that the swamp work, rather than more typical exposures, put residents at increased risk for malaria. In this community, anecdotally adults tend to work in the field until late evening, which might expose them to more mosquito bites. Adults also tend to engage in outdoor activities more and for longer hours, and they usually stayed up later than children.

Our investigation highlighted the reality that, as countries work towards malaria control and elimination, malaria control efforts could be hindered and rapidly reversed, and traditionally low-risk areas can become high-risk areas. Factors that could hinder progress towards malaria control and elimination include changes in macro conditions due to climate change, such as temperature and precipitation, that promote mosquito breeding. They can also be factors that are caused by human activities such as expansion of agricultural activities near residential areas that increase areas of standing water, such as new rice paddy [32] and other crop fields, and brick-making, as observed in the current investigation. Also, as transmission declines, residents may become complacent and stop using protective measures such as larviciding of breeding sites, and use of insecticide treated bednets and mosquito repellents. Malaria control programs should therefore be vigilant about these challenges, strengthen the surveillance system to detect outbreaks early, and reevaluate and refocus control activities so as to ensure continued progress towards elimination. 
Our investigation had several limitations. We were unable to discern between multiple positive malaria tests by the same person versus unique positive tests for village residents. This may have led to an overestimation of the attack rates. The use of neighborhood control might have caused overmatching in the case-control study, especially for the effect of "having a swamp nearby". Also, during the entomological assessment, no quantitative measurement was conducted on the density of various stages of Anopheles mosquitos in the trenches of the rice paddy, Irish-potato, and brick-making fields.

\section{Conclusions}

Increased human activities, including agriculture and other activities (such as Irish potato growing, rice farming, and brick-making) facilitated malaria transmission among residents in an area previously reporting low malaria transmission. Lack of bed-net use and inadequate protection against mosquito bites facilitated transmission of malaria during the outbreak.

\section{Recommendations}

We recommended increased awareness about bed net usage, and use of larvicides to reduce mosquito density in the area. Additionally, health education should be conducted on protective behavior to avoid mosquito bites, such as wearing of long protective clothes in the late evenings and while engaged in outdoor late activities.

\section{List Of Abbreviations}

AR: Attack rate; $\mathrm{Cl}$ : Confidence Interval; $\mathrm{UMOH}$ : Uganda Ministry of Health; $\mathrm{OR}_{\mathrm{CLR}}$ : Odds ratio estimated from conditional logistic regression.

\section{Declarations}

\section{Acknowledgements}

We would like to thank Annet Joselyn Atuhairwe for her technical guidance during our entomological assessment. We thank Kisoro District Local Government for the cooperation during the investigation and the Village Health Teams for the guidance in the community during data collection.

\section{Funding}

This project was funded by the Cooperative Agreement - Provision of Comprehensive HIV/AIDS services and Developing National Capacity to manage HIV/AIDS Programs in the Republic of Uganda under the President's Emergency Plan for AIDS Relief (grant number 5U2GGH000817-03), through the United States Centers for Disease Control and Prevention, awarded to the Makerere University School of Public Health to support the Uganda Public Health Fellowship Program, $\mathrm{MOH}$ of Uganda. The funders had no role in study design, data collection and analysis, decision to publish, or preparation of the manuscript. 


\section{Availability of data and materials}

The datasets generated during and/or analysed during the current study are not publicly available due to confidentiality conditions. The authors agreed not to reproduce or distribute them.

\section{Disclaimer}

The views and contents herein are solely those of the authors and do not necessarily represent the official views of the United States Centers for Disease Control and Prevention, Makerere University School of Public Health, or the Ministry of Health Uganda.

\section{Authors' contributions}

JN, OD investigated the outbreak under technical guidance and supervision of DK and B-PZ. JN, OD, DK, ARA, and B-PZ analysed, interpreted the data and drafted the manuscript. ARA, DK, LB and B-PZ critically reviewed the manuscript for intellectual content. All co-authors read and approved the final manuscript. JN is the guarantor of the paper.

\section{Ethics approval and consent to participate}

This investigation was in response to a public health emergency and was therefore determined to be nonresearch. The $\mathrm{MOH}$ gave the directive and approval to investigate this outbreak. The Office of the Associate Director for Science, Center for Global Health, CDC, determined that this activity was not human subject research, and its primary intent was public health practice or a disease control activity. Verbal informed consent in the local language was sought from respondents or caretakers of diseased children.

\section{Consent for publication}

Not applicable.

\section{Competing interests}

We declare that there are no competing interests.

\section{References}

1. World Health Organization, Malaria fact sheet. 2017: Geneva, Switzerland.

2. Protopopoff, N., et al., Ranking malaria risk factors to guide malaria control efforts in African highlands. PLoS One, 2009. 4(11): p. e8022.

3. Koram, K., et al., Socio-economic risk factors for malaria in a peri-urban area of The Gambia. Transactions of the royal society of tropical medicine and hygiene, 1995. 89(2): p. 146-150. 
4. Lindblade, K.A., et al., Highland malaria in Uganda: prospective analysis of an epidemic associated with El Nino. Transactions of the Royal Society of Tropical Medicine and Hygiene, 1999. 93(5): p. 480-487.

5. Lindblade, K.A., et al., Land use change alters malaria transmission parameters by modifying temperature in a highland area of Uganda. Tropical Medicine \& International Health, 2000. 5(4): p. 263-274.

6. O'Meara, W.P., et al., Changes in the burden of malaria in sub-Saharan Africa. The Lancet infectious diseases, 2010. 10(8): p. 545-555.

7. Barber, B.E., et al., World Malaria Report: time to acknowledge Plasmodium knowlesi malaria. Malaria journal, 2017. 16(1): p. 135.

8. Geneva, W. and W.H. Organization, Malaria elimination: a field manual for low and moderate endemic countries. 85. 2007.

9. Yeka, A., et al., Malaria in Uganda: challenges to control on the long road to elimination: I. Epidemiology and current control efforts. Acta tropica, 2012. 121(3): p. 184-195.

10. Okello, P.E., et al., Variation in malaria transmission intensity in seven sites throughout Uganda. The American journal of tropical medicine and hygiene, 2006. 75(2): p. 219-225.

11. Curtis, C., et al., Insecticide-treated bed-nets for malaria mosquito control. Journal of the American Mosquito Control Association, 2006. 22(3): p. 501-506.

12. Galactionova, K., et al., Effective coverage and systems effectiveness for malaria case management in subSaharan African countries. PLoS One, 2015. 10(5): p. e0127818.

13. Okullo, A.E., et al., Malaria incidence among children less than 5 years during and after cessation of indoor residual spraying in Northern Uganda. Malaria journal, 2017. 16(1): p. 319.

14. Programme., N.M.C., Uganda Malaria Reduction Strategic Plan 2014-2020.Kampala. 2014.

15. Statistics, U.B.o. and O.M. MEASURE/DHS+, Uganda Demographic and Health Survey, 2006. 2007: Uganda Bureau of Statistics.

16. Uganda Ministry of Health, District Health Information System (DHIS2) dashboard. Ministry of Health, 2018.

17. Uganda, M., District Health Information System (DHIS2) dashboard. Ministry of Health, 2018.

18. Uganda Bureau of Statistics (UBOS), Ugand National Population and Housing Census. 2014.

19. TAYLOR, B., Changes in the feeding behaviour of a malaria vector, Anopheles farauti Lav., following use of DDT as a residual spray in houses in the British Solomon Islands Protectorate. Ecological Entomology, 1975. 127(3): p. 277-292.

20. Wirtz, R., et al., ELISA method for detecting Plasmodium falciparum circumsporozoite antibody. Bulletin of the World Health Organization, 1989. 67(5): p. 535. 
21. Fontaine, R.E., Describing Epidemiologic Data, in The CDC Field Epidemiology Manual, S.A. Rasmussen and R.A. Goodman, Editors. 2019, Oxford University Press: New York.

22. Bayoh, M.N., et al., Anopheles gambiae: historical population decline associated with regional distribution of insecticide-treated bed nets in western Nyanza Province, Kenya. Malaria journal, 2010. 9(1): p. 62.

23. Kureya, T., A. Ndaimani, and M. Mhlanga, Malaria Outbreak Investigation in Chipinge, Zimbabwe: A Casecontrol Study. Iranian journal of parasitology, 2017. 12(3): p. 423.

24. Mugwagwa, N., et al., Factors associated with malaria infection in Honde valley, Mutasa district, Zimbabwe, 2014: a case control study. BMC Research Notes, 2015. 8(1): p. 829.

25. Chiruvu RT, K.B., Mungati M, et al. 2015. , Malaria Outbreak investigation in Chitulipasi, Beitbridge District, Matabeleland South Province,2015. IJRDO-Journal of Health Sciences and Nursing., 2017.2 ((5):): p. $104-120$.

26. Lindblade, K.A., et al., A cohort study of the effectiveness of insecticide-treated bed nets to prevent malaria in an area of moderate pyrethroid resistance, Malawi. Malaria Journal, 2015. 14(1): p. 31.

27. Krishnamoorthy, K., et al., Altered environment and risk of malaria outbreak in South Andaman, Andaman \& Nicobar Islands, India affected by tsunami disaster. Malaria journal, 2005. 4(1): p. 32.

28. Hay, S.I., et al., The global distribution and population at risk of malaria: past, present, and future. The Lancet infectious diseases, 2004. 4(6): p. 327-336.

29. Sinton, J.A., 'Man-Made' Malaria in India. The Indian Medical Gazette, 1936. 71(4): p. 181.

30. Gil, L.H.S., et al., Urban and suburban malaria in Rondônia (Brazilian Western Amazon) II: perennial transmissions with high anopheline densities are associated with human environmental changes. Memórias do Instituto Oswaldo Cruz, 2007. 102(3): p. 271-276.

31. Keiser, J., B.H. Singer, and J. Utzinger, Reducing the burden of malaria in different eco-epidemiological settings with environmental management: a systematic review. The Lancet infectious diseases, 2005. 5(11): p. 695-708.

32. ljumba, J., S.J.M. Lindsay, and v. entomology, Impact of irrigation on malaria in Africa: paddies paradox. 2001. 15(1): p. 1-11.

\section{Tables}

Table 1: Age-specific attack rates of malaria during an outbreak: Kisoro District, Uganda, November 2017-May 2018 


\begin{tabular}{lrrc}
\hline Variable & Frequency & Population & AR/1000 \\
\hline Sex & & & \\
$\quad$ Male & 2317 & 126,055 & 18 \\
$\quad$ Female & 2501 & 155,650 & 16 \\
Age (years) & & & \\
$0-9$ & 1350 & 101,350 & 13 \\
$10-19$ & 1454 & 76,720 & 19 \\
$20-29$ & 908 & 44,480 & 20 \\
$30-39$ & 527 & 29,050 & 18 \\
$40-49$ & 286 & 19,480 & 15 \\
$50-59$ & 206 & 14,430 & 14 \\
$\geq 60$ & 87 & 16,290 & 05.3 \\
\hline
\end{tabular}

able 2: Exposures associated with malaria infection during an outbreak: Kisoro District, Uganda, November 2017 ग18

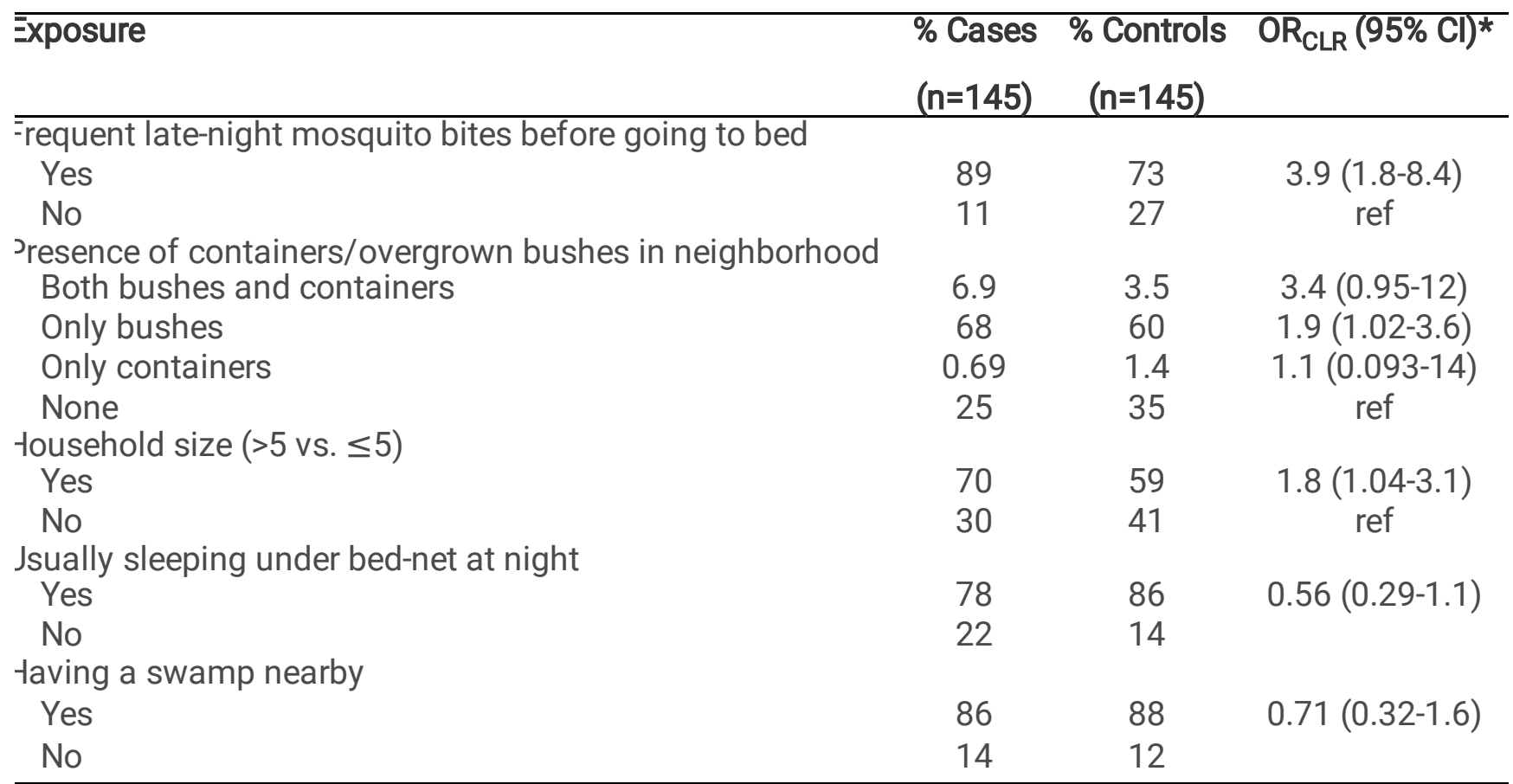

* Odds ratio and $95 \%$ confidence interval using conditional logistic regression, using matched set as the matching variable.

\section{Figures}




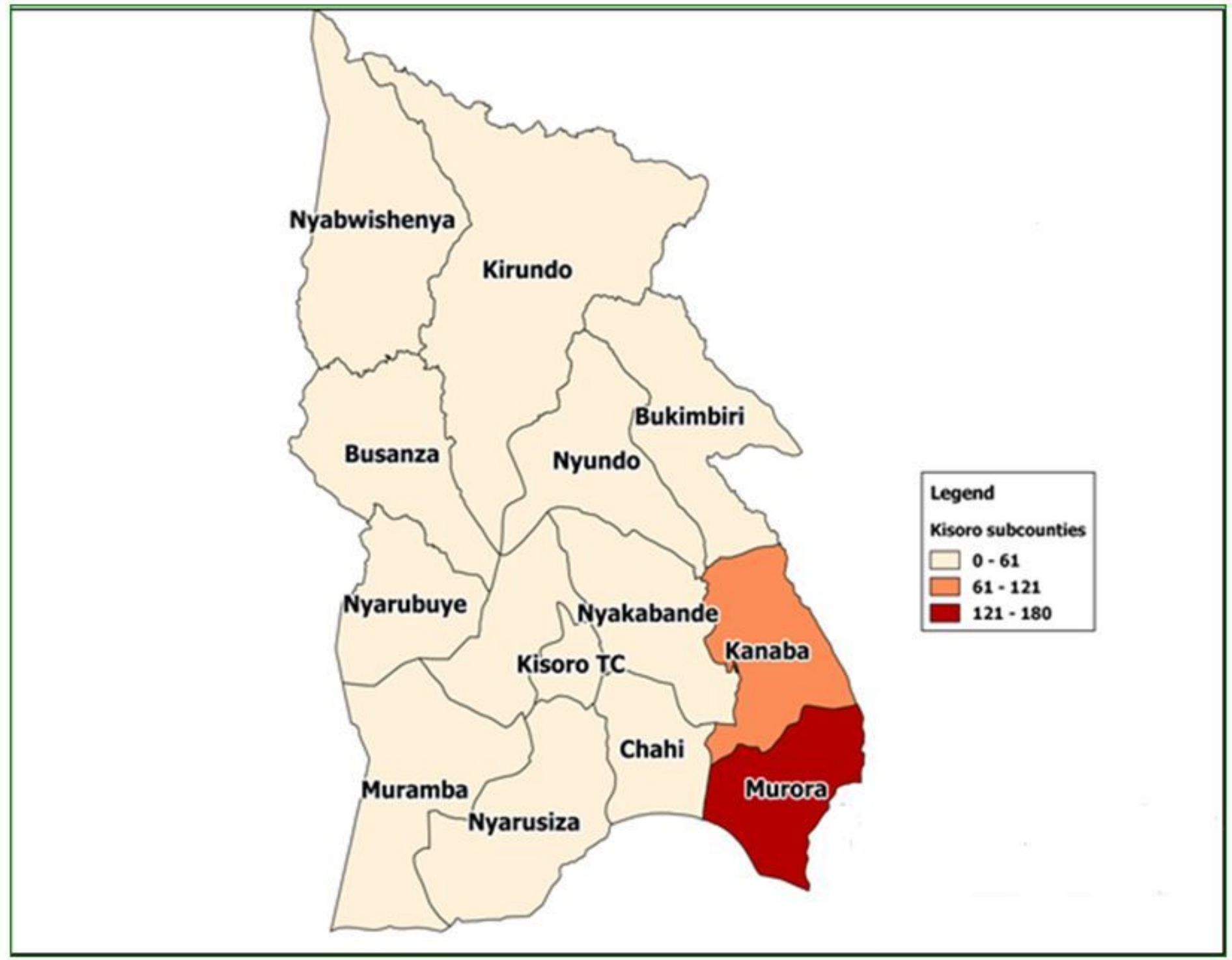

Figure 1

Attack rate $(/ 1,000)$ by sub-county during a malaria outbreak: Kisoro District, Uganda, November 2017-May 2018 


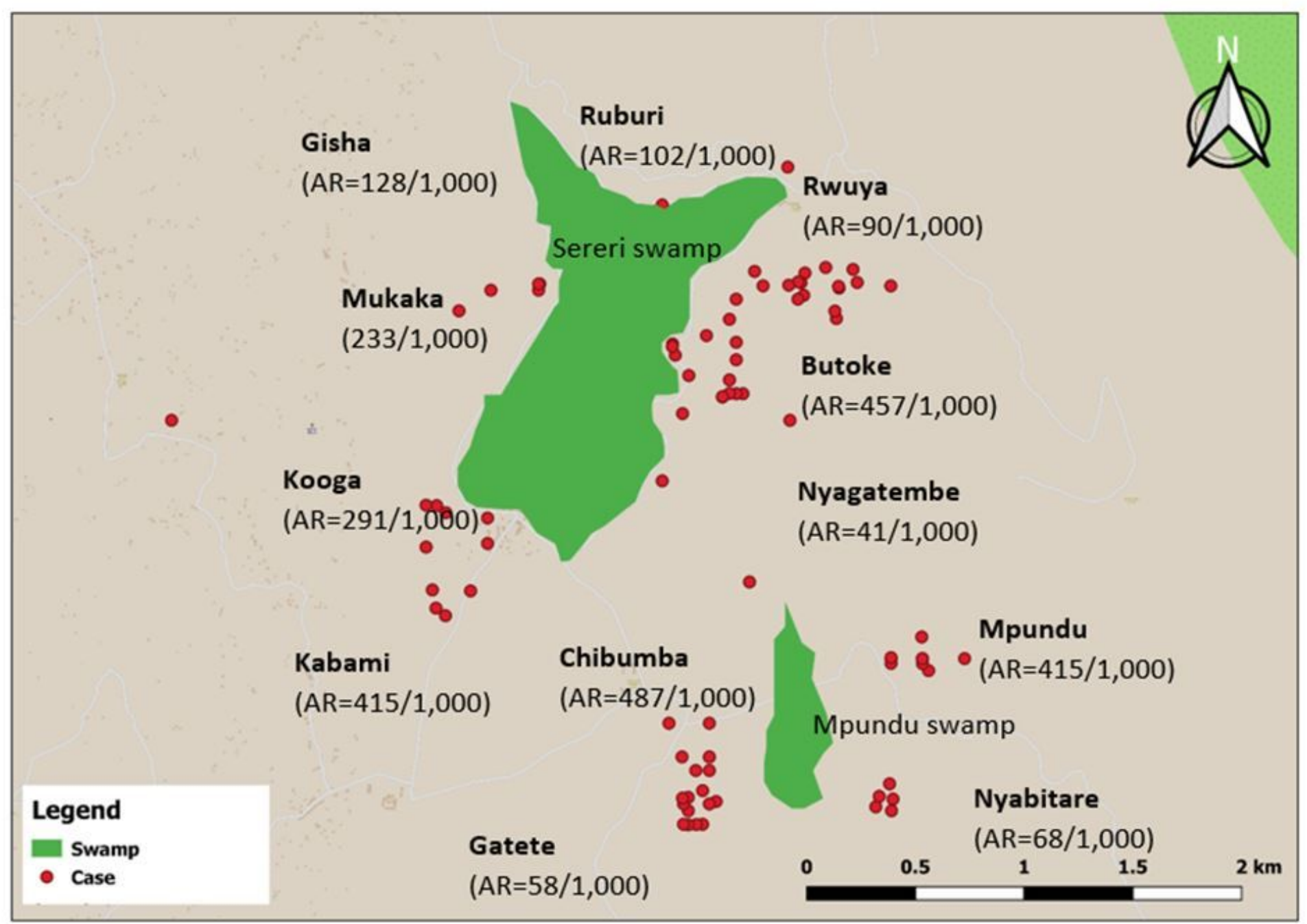

Figure 2

Distribution of cases used in the case-control study (selected using systematic randomly sampling from the line list) around Sereri and Mpundu swamps during a malaria outbreak: Kisoro District, Uganda, December 2017March 2018 


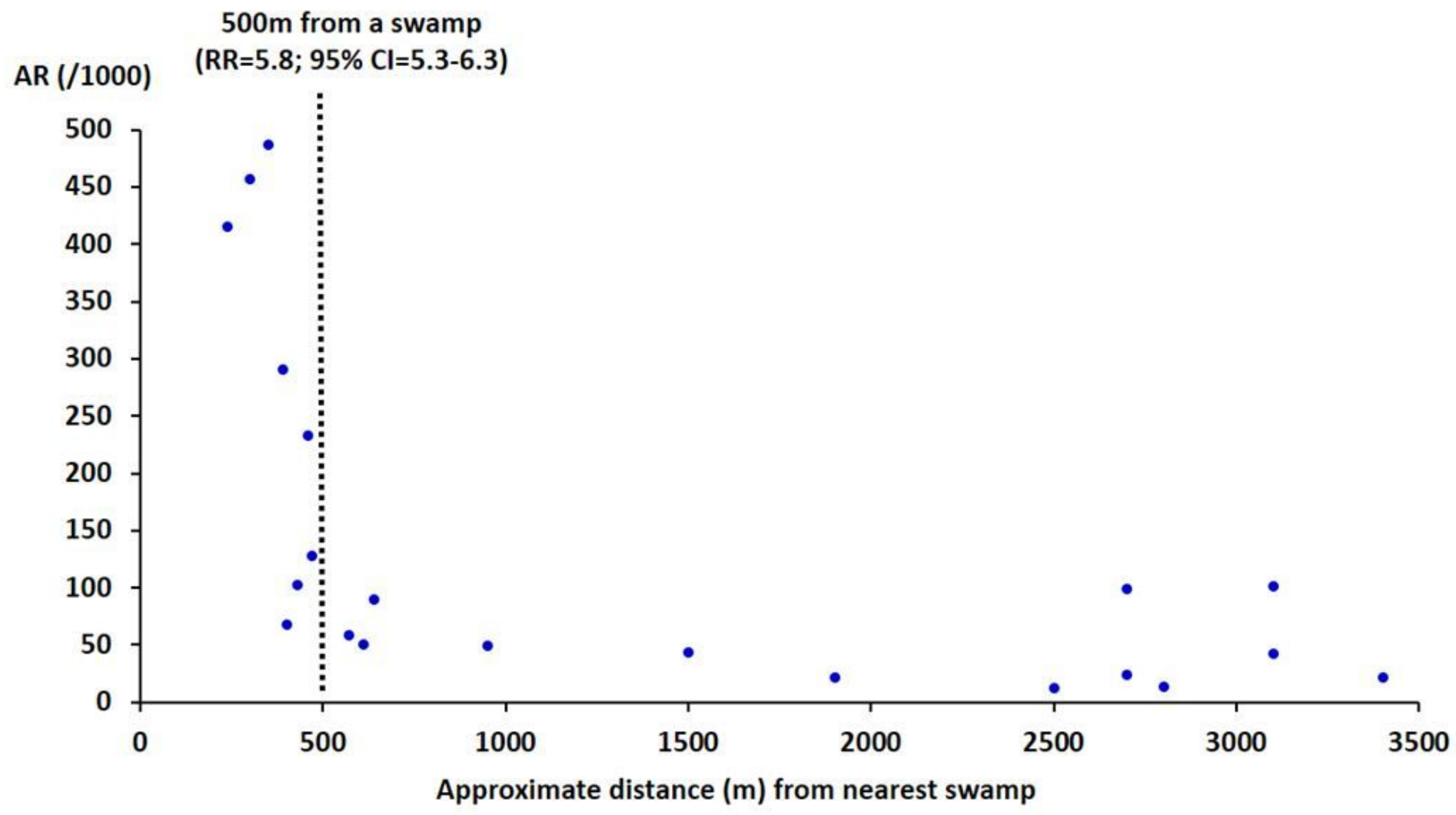

\section{Figure 3}

Attack rates (\%) in villages located around Sereri and Mpundu swamps during a malaria outbreak: Kisoro District, Uganda, December 2017-March 2018

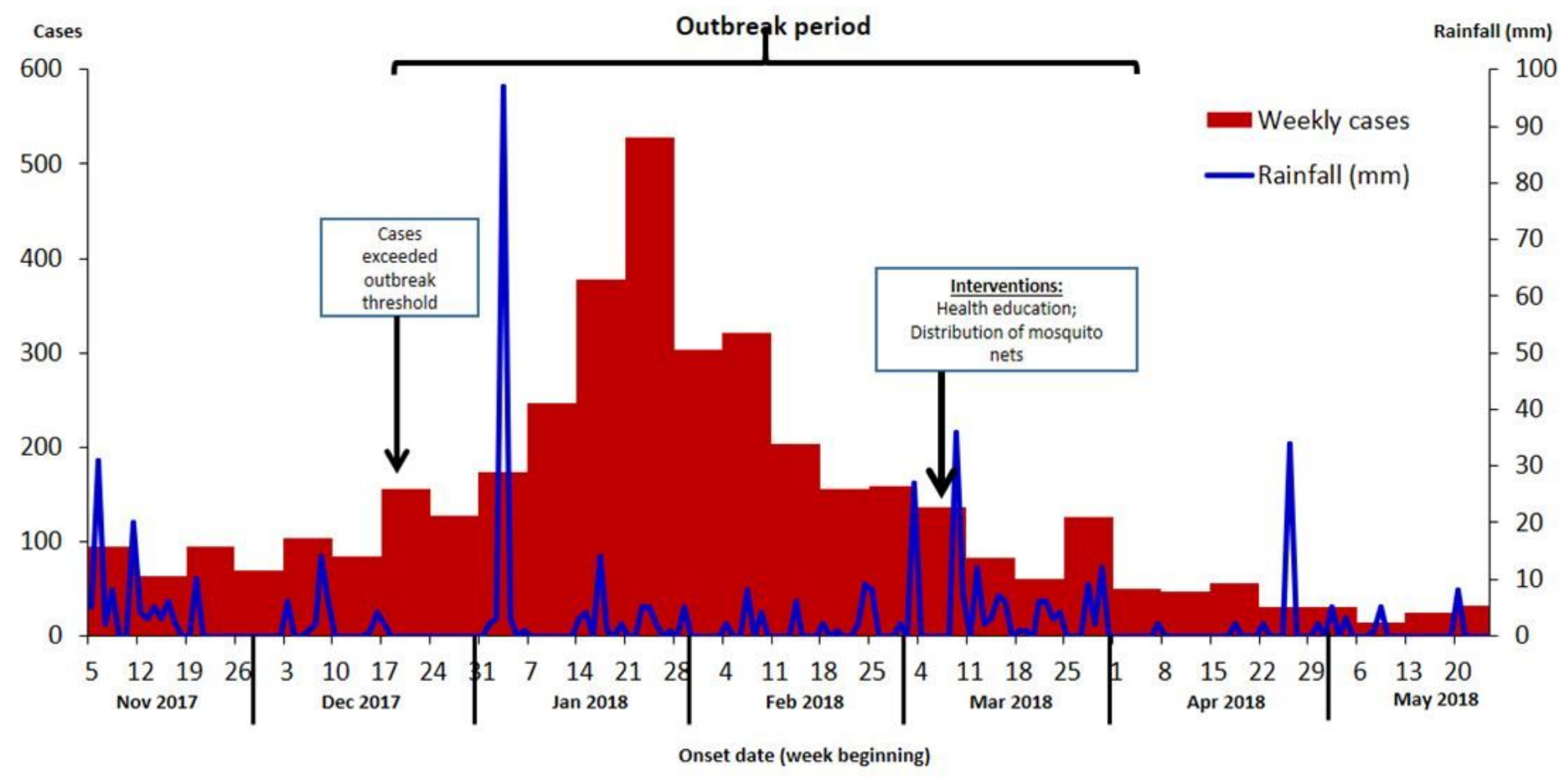

Figure 4 
Week of symptom onset of cases and rainfall amount during a malaria outbreak: Kanaba and Murora Subcounties, Kisoro District, November 2017-May 2018 